\title{
Loops of Humans and Bots in Wikidata*
}

\author{
Elena Simperl ${ }^{\dagger}$ \\ University of Southampton \\ Southampton, United Kingdom \\ E.Simperl@soton.ac.uk
}

\begin{abstract}
Wikidata is one of most successful knowledge graphs ever created. It expresses knowledge in the form of subject-property-value statements accompanied by provenance information. A project of the Wikimedia Foundation, Wikidata is supported by a community of currently 19 thousand active users and 234 bots, who together are responsible for editing more than 45 million entities since the start of the project in 2012. This makes Wikidata a prime example for what human-in-the-loop technology can achieve. In this talk, we are going to present several studies that aim to understand the links between its socio-technical fabric and its success.
\end{abstract}

\section{CCS CONCEPTS}

- Human-centered computing $\rightarrow$ HCI design and evaluation methods; HCI design and evaluation methods; $\bullet$ Information systems $\rightarrow$ World Wide Web; • Theory of computation $\rightarrow$ Semantics and reasoning;

\section{KEYWORDS}

wikidata; humans in the loop; socio-technical fabric

ACM Reference Format:

Elena Simperl. 2018. Loops of Humans and Bots in Wikidata. In The 2018 Web Conference Companion, April 23-27, 2018, Lyon, France. ACM, New York, NY, USA, 1 page. https://doi.org/10.1145/3184558.3191552

${ }^{*}$ Loops of Humans and Bots in Wikidata

${ }^{\dagger}$ This is the corresponding author

This paper is published under the Creative Commons Attribution 4.0 International (CC BY 4.0) license. Authors reserve their rights to disseminate the work on their personal and corporate Web sites with the appropriate attribution.

WWW'18 Companion, April 23-27, 2018, Lyon, France

(c) 2018 IW3C2 (International World Wide Web Conference Committee), published under Creative Commons CC BY 4.0 License.

ACM ISBN 978-1-4503-5640-4/18/04.

https://doi.org/10.1145/3184558.3191552 